\title{
Caracterização de riscos em protocolos submetidos a um comitê de ética em pesquisa: análise bioética
}

Camila Maria Pereira Rates ${ }^{1}$, Marcella Rodrigues e Costa ${ }^{2}$, Juliana Dias Reis Pessalacia ${ }^{3}$

\section{Resumo}

Estudo descritivo realizado com o objetivo de identificar como os pesquisadores descreveram os riscos e as medidas de proteção/prevenção de suas pesquisas submetidas à análise por um comitê de ética em pesquisa. Foram incluídos 175 protocolos de pesquisa de um comitê de ética em pesquisa da cidade de Divinópolis, Minas Gerais. Como resultado, encontrou-se que somente 38 (24,7\%) pesquisadores descreveram os riscos nos formulários e 36 (23,5\%) no termo de consentimento. Entretanto, 110 (62,9\%) descreveram as medidas de prevenção nos formulários e 143 (93,5\%) as descreveram no termo de consentimento. Concluiu-se que, embora os pesquisadores tenham identificado as medidas de prevenção, não descreveram quais seriam propriamente os riscos de suas pesquisas. É possível que tenham certa dificuldade em descrever os riscos por associá-los a danos.

Palavras-chave: Comitê de revisão ética. Ética em pesquisa. Protocolos. Bioética.

\section{Resumen}

Caracterización de los riesgos en los protocolos sometidos a un comité de ética en investigación: análisis bioético

Estudio descriptivo con el fin de conocer cómo los investigadores describieron los riesgos y las medidas de protección/prevención de sus investigaciones sometidas a análisis por un comité de ética en investigación. Se evaluaron 175 protocolos de investigación de un comité de ética en investigación de la ciudad de Divinópolis, Minas Gerais. Como resultado sólo 38 (24,7\%) de los investigadores describieron los riesgos en los formularios y $36(23,5 \%)$ en el término de consentimiento informado. Sin embargo, 110 (62,9\%) describieron las medidas de prevención en los formularios y $143(93,5 \%)$ en el término de consentimiento informado. Se concluye que aunque los investigadores han identificado las medidas de prevención, no describieron exactamente cuáles son los riesgos de sus investigaciones. Es posible que tengan alguna dificultad en la descripción de los riesgos por asociarlos a daños.

Palabras-clave: Comité de revisión ética. Ética en la investigación. Protocolos. Bioética.

\section{Abstract \\ Characterization of risks in search of an ethics committee in research protocols: bioethical analysis}

A descriptive study in order to learn how researchers described risks and protective/preventive measures of their researches submitted to analyzes by an ethics committee in research. 175 research protocols submitted to a research ethics committee were included. As a result, only 38 (24.7\%) described the risks in the forms and $36(23.5 \%)$ in the informed consent. However, 110 (62.9\%) described preventive measures in the forms and $143(93.5 \%)$ in the informed consent. It was concluded that, although researchers have identified preventive measures, they have not described the risks to which the measures were targeted. It is possible that they find it hard to describe risks since they relate them to injuries.

Keywords: Ethical review committee. Ethics in research. Protocols. Bioethics.

Aprovação CEP Hospital São João de Deus 183/11

1. Mestranda camila.rates@yahoo.com.br 2. Graduanda marcella_nany@hotmail.com 3. Doutora juliana@pessalacia.com.br Universidade Federal de São João Del Rei (UFSJ), Campus Centro-Oeste Dona Lindu, Divinópolis/MG, Brasil.

Juliana Dias Reis Pessalacia - Rua São Paulo, 1.710/104 Santo Antônio CEP 35502-025. Divinópolis/MG, Brasil. 
As pesquisas realizadas por profissionais de saúde envolvem, muitas vezes, a participação de seres humanos, fato que torna imprescindível a avaliação do projeto de pesquisa por um comitê de ética independente, antes de sua realização. A avaliação ética realizada pelos comitês de ética em pesquisa (CEP) com seres humanos prevê que uma pesquisa somente será justificável se os seus benefícios sobrepujarem seus riscos e se não houver risco iminente de morte ou invalidez para os sujeitos participantes. Além disso, devem-se estabelecer medidas de prevenção ou minimização dos riscos identificados na pesquisa.

A Resolução 466/12, homologada pelo ConseIho Nacional de Saúde (CNS) do Ministério da Saúde (MS), afirma que toda pesquisa com seres humanos envolve riscos os quais devem ser previstos e descritos no protocolo de pesquisa a ser avaliado pelo CEP. Ainda define, em seu inciso II-22: Risco da pesquisa: possibilidade de danos à dimensão física, psíquica, moral, intelectual, social, cultural ou espiritual do ser humano, em qualquer fase de uma pesquisa $e$ dela recorrente ${ }^{1}$.

Dessa forma, é importante que o pesquisador tenha consciência ética e saiba fazer uma análise crítica dos riscos de suas pesquisas. Entende-se que a ética tem o propósito de refletir sobre o agir humano e suas finalidades, a fim de compreender os critérios e valores que orientam o julgamento da ação em suas diversas atividades ${ }^{2}$.

A bioética - campo que pode auxiliar a tomada de decisão, assim como a reflexão acerca dos riscos de uma pesquisa - pode ser definida como o estudo sistemático do agir humano na área das ciências da vida e dos cuidados da saúde, na medida em que essa conduta é examinada à luz dos valores e princípios morais ${ }^{3} \mathrm{~A}$ bioética se apresenta como uma tentativa de apreender e compreender o verdadeiro significado do novo, capacitando-nos a uma provável adaptação ${ }^{4}$.

Assim como qualquer atividade humana, a pesquisa contém variadas dimensões éticas ${ }^{5}$. O termo "pesquisa" se relaciona com uma classe de atividades cujo objetivo é desenvolver ou contribuir para o conhecimento generalizável, o qual consiste em teorias, princípios ou relações ${ }^{6}$. Em grande parte dos países democráticos contemporâneos, existe a percepção de que toda pesquisa conduzida em qualquer área do conhecimento, e que envolva seres humanos como objetos da investigação, deve ser revisada em âmbito epistemológico, metodológico e ético, por seus pares e pelo conjunto da sociedade por intermédio de instância competente e reconhecida ${ }^{7}$.
A ideia da necessidade de controle dos experimentos para assegurar o bem-estar dos participantes surgiu com o Código de Nüremberg, de 1947, que determina a necessidade do consentimento voluntário dos indivíduos envolvidos na pesquisa, após o seu devido esclarecimento sobre os objetivos e os riscos do projeto. Esse documento influenciou a criação pela Associação Médica Mundial (AMA), em 1964, da Declaração de Helsinki, que refina e corrobora parâmetros éticos para estudos conduzidos por profissionais de medicina. Esta declaração sofreu algumas modificações entre as décadas de 1970 e 1990. Em 1975, foi incorporada a obrigatoriedade de aprovação prévia de qualquer projeto de pesquisa em seres humanos por um CEP ${ }^{8}$.

No Brasil, desde 1988 o CNS já havia deliberado pela formação de CEP para estudos com seres humanos mediante a Resolução $1 / 88$, marco importante no trajeto de formalização da ética em pesquisa no campo da saúde. Tal resolução foi revista em 1995, quando nascia a bioética pública, responsável pelo desafio de enfrentar os novos problemas sanitários que se consolidavam na última década do século $X X$. Entre outras tantas atribuições, ao CNS coube um novo papel: o acompanhamento do processo de desenvolvimento e incorporação científica e tecnológica na área de saúde. Surge, assim, a Resolução 196, de 16 de outubro de 1996, instituindo o Sistema CEP/Comissão Nacional de Ética em Pesquisa (Conep) para avaliação de pesquisas envolvendo seres humanos, o qual, além dos CEP locais, passou a contar com a Conep para análise de tipos específicos de estudo. O Sistema CEP/Conep foi espelho de um processo que contemplou, com grande margem de detalhamento, as exigências éticas destinadas a nortear a pesquisa no campo da saúde ${ }^{9}$. A Resolução 196/96 passou por revisão e originou a Resolução $466 / 12$, norma hoje vigente que mantém muitos pontos da resolução anterior.

Uma das exigências dispostas na Resolução 196/96, e mantida na Resolução 466/12, refere-se ao termo de consentimento livre e esclarecido (TCLE). Trata-se da aprovação dada pelo sujeito e/ ou por seu representante legal, após explicação completa sobre a natureza da pesquisa, seus objetivos, métodos, benefícios previstos, potenciais riscos e o incômodo que ela possa acarretar, formulada em um termo de consentimento, autorizando sua participação voluntária na pesquisa ${ }^{10}$. A norma considera que, potencialmente, todo experimento pode provocar danos permanentes ou eventuais de natureza física, psicológica, social, moral, intelectual, cultural, espiritual e econômica ${ }^{6}$. Assim, o presente 
estudo teve o objetivo de identificar como os pesquisadores descreveram os riscos e as medidas de proteção/prevenção de suas pesquisas submetidas à análise por um CEP.

\section{Método}

Tratou-se de um estudo do tipo descritivo e exploratório, realizado a partir da coleta de dados preexistentes em um CEP com seres humanos de um hospital filantrópico de Divinópolis, Minas Gerais, Brasil. Trata-se de um CEP registrado na Conep, o qual desde 2005 realiza reuniões mensais para análise de seus projetos. Para a coleta de dados, utilizou-se um roteiro contendo questões fechadas acerca da caracterização das pesquisas, riscos e medidas de proteção, sendo, portanto, definidas a priori as categorias do estudo. Tal roteiro foi elaborado pelos próprios pesquisadores, com base em dados encontrados na literatura sobre a temática.

Foram incluídos neste estudo todos os protocolos de pesquisa dos pesquisadores que submeteram seus projetos ao CEP, no período de março de 2006 a março de 2011, independentemente de seu parecer final, isto é, se foi aprovado, não aprovado ou ficou pendente. Cabe dizer que foram excluídos os protocolos ilegíveis e/ou com informações incompletas. Dessa forma, a amostra final constituiu-se em 175 protocolos.

Os dados coletados foram dispostos em um banco de dados criado no Microsoft Excel e, posteriormente, analisados por meio de estatística descritiva simples. Para a preservação dos aspectos éticos da pesquisa com seres humanos, foram seguidas as disposições da Resolução 466/12 e, antes da coleta de dados, o projeto de pesquisa foi submetido à análise pelo CEP da instituição pesquisada.

\section{Resultados e discussão}

Sobre a categoria profissional dos pesquisadores, observou-se que 75 (42,3\%) eram enfermeiros; 58 (33,2\%), médicos; 12 (6,9\%), fisioterapeutas; 10 (5,7\%), farmacêuticos; 8 (4,6\%), biólogos; e 12 (7,3\%), outros profissionais. A predominância de enfermeiros entre os pesquisadores que submeteram seus protocolos de pesquisa ao CEP pode estar relacionada com o atual incremento da pesquisa na área de enfermagem. Além disso, nota-se, recentemente, grande aumento da divulgação de pesquisas realizadas por enfermeiros ${ }^{11}$.
Com relação ao vínculo institucional dos pesquisadores, encontrou-se que a maioria, isto é, 96 $(54,9 \%)$, trabalha em universidades; $57(32,5 \%)$ trabalham em hospitais; 15 (8,6\%), em outros locais; e 7 (4\%) não trabalham. Estudo realizado a fim de traçar e analisar o perfil do CEP da Universidade da Amazônia também apontou que, embora exista diversidade considerável no que diz respeito à profissão dos pesquisadores, há presença marcante de professores universitários entre os profissionais ${ }^{12}$. Considerando que as bases da pesquisa estão na formação acadêmica, pode-se dizer que a predominância de professores universitários (54,9\%) entre os pesquisadores está diretamente relacionada com a necessidade de atuação conjunta entre ensino e pesquisa.

Quanto à situação final da análise dos protocolos após primeira avaliação realizada pelos pareceristas, notou-se que $16(9,1 \%)$ foram reprovados, 22 (12,6\%) foram aprovados e 137 (78,3\%) ficaram pendentes. Pesquisa desenvolvida com o objetivo de relatar a experiência do CEP da Secretaria de Estado de Saúde (SES) do Distrito Federal (DF), Brasil, durante 10 anos, a partir de sua fundação, verificou que o baixo percentual de protocolos não aprovados pode ser entendido como reflexo de ações educativas promovidas pelo comitê ${ }^{13}$. Outro estudo conduzido na Escola de Enfermagem (EE) da Universidade de São Paulo (USP) também apontou que, dos 399 projetos apreciados, 232 (58,2\%) foram aprovados com pendência e apenas 5 (1,3\%) foram reprovados ${ }^{14}$.

Figura 1. Motivos das pendências presentes nos protocolos de pesquisa analisados por um CEP do município de Divinópolis/MG, Brasil ( $n=175)$

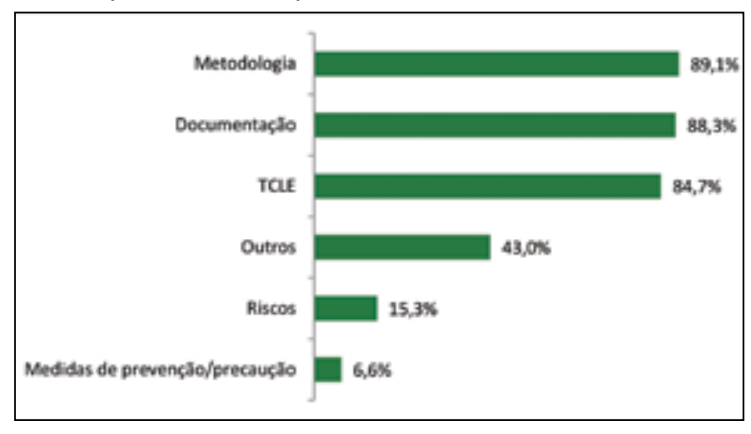

A Figura 1 mostra que a maioria dos protocolos $(89,1 \%)$ consistia em pendências referentes à metodologia. É importante notar que no item "Outros" foram incluídos aqueles protocolos que apresentaram pendências relacionadas com informações incompletas, erros no orçamento, critérios de inclusão e exclusão, abrangendo $43 \%$ dos protocolos. 
Cabe salientar que em todos os protocolos pendentes constavam dois ou mais motivos de pendência.

Pesquisa realizada por outros autores ${ }^{12}$ também aponta muitas das pendências identificadas nos protocolos de pesquisa incluídos neste estudo, uma vez que as pendências mais frequentemente encontradas nos protocolos avaliados pelo colegiado do CEP referem-se à solicitação de reavaliação do TCLE (30\%); informações incompletas na folha de rosto (25\%); ausência de informações sobre o tipo de estudo na metodologia: amostragem, critérios de inclusão e exclusão, forma de alocação e análise dos dados (20\%); planilha de orçamento ausente ou incompleta (12\%); curriculum vitae de todos os pesquisadores citados: incompleto ou ausente (9\%); outros fatores (4\%).

\section{Descrição dos riscos nos protocolos de pesquisa}

Quanto à descrição dos riscos no protocolo, dos 175 pesquisadores, 88 (57,1\%) classificaram seus projetos como isentos de risco e 66 (42,9\%) avaliaram como de risco mínimo, entre esses 28 $(42,4 \%)$, embora tenham classificado seus projetos como tendo risco de grau mínimo, alegaram nos aspectos éticos que a pesquisa não ofereceria riscos aos sujeitos, já que se tratava apenas de um questionário/entrevista. Tal discrepância permite pressupor que entre os pesquisadores existe a tendência de minimizar os possíveis riscos dos estudos.

Com relação aos outros 38 projetos que continham descrição dos riscos nos aspectos éticos $(57,6 \%)$, foram identificadas duas modalidades de risco nas descrições: riscos de origem psicológica, intelectual ou/e emocional, bem como riscos de ordem física e orgânica. Entre os primeiros foram encontrados: a possibilidade de constrangimento ao responder o questionário; desconforto; estresse; quebra de sigilo; dano; cansaço ao responder às perguntas; e quebra de anonimato. Quanto ao segundo tipo, foram especificados os seguintes riscos: sangramento, dor e até risco de morte. Cabe dizer que, entre os protocolos de pesquisa analisados, 21 (12\%) não continham o formulário de encaminhamento, o que impossibilitou avaliar como os pesquisadores classificaram e descreveram os riscos de suas pesquisas.

Percebe-se que, entre os pesquisadores, é comum classificar os riscos de uma pesquisa como sendo inexistentes. Colabora, para tal percepção, um estudo desenvolvido com a finalidade de conhecer os riscos na utilização de questionários/entrevistas, segundo o qual 18 pesquisadores também disseram não existir nenhum tipo de risco em suas pesquisas com questionários ou entrevistas. No entanto, os autores do estudo enfatizaram que tais instrumentos podem ser considerados como possíveis causadores de danos, desconfortos e constrangimentos quando há falta de cuidado na elaboração do conteúdo e no modo de aplicação. Também observaram que, dependendo do tipo de questão, a aplicação do questionário pode provocar níveis incomuns de constrangimento, causando experiências negativas ${ }^{15}$.

Descrição das medidas de precaução/prevenção nos protocolos de pesquisa

Sobre o apontamento das medidas de precaução/prevenção documentadas pelo pesquisador em seus projetos de pesquisa, observou-se que 65 $(37,1 \%)$ não apontaram tais medidas, ao passo que $110(62,9 \%)$ as identificaram das seguintes formas: as respostas serão confidenciais; o questionário não será identificado pelo nome para que seja mantido o anonimato; os indivíduos receberão esclarecimento prévio sobre a pesquisa; a entrevista poderá ser interrompida a qualquer momento; leitura do TCLE, autorização legal quando sujeito for vulnerável, assistência psicológica se necessária; privacidade para responder o questionário; garantia de sigilo; participação voluntária e consideração de situação de vulnerabilidade, quando houver.

Portanto, entre os pesquisadores, embora apenas $38(20,1 \%)$ tenham descrito os riscos de suas pesquisas nos protocolos, 110 (62,9\%) apontaram as medidas de precaução/prevenção. Notou-se ainda que os pesquisadores conseguem identificar as medidas de precaução/prevenção, mas têm certa dificuldade em apontar os riscos. É possível perceber, na análise dos protocolos, a forte associação entre risco e dimensão física, evidenciada pelo fato de que a maior parte das descrições identifica prejuízos de natureza orgânica. Entretanto, circunscrever risco a essa dimensão é reducionismo, é o mesmo que não perceber o ser humano como indivíduo social, com valores, cultura, crenças e emoções. O impacto proveniente do uso de instrumentos de pesquisa não invasivos no corpo físico, tais como questionários ou entrevistas, frequentemente não é considerado; logo, os danos não são inteiramente explorados ${ }^{15}$.

Ainda que não sejam feitas em seres humanos e sim com seres humanos, as pesquisas qualitativas podem causar danos ou trazer prejuízo àqueles que participam do estudo, e essa possibilidade precisa ser avaliada com o mesmo cuidado que se emprega nos ensaios que afetam a dimensão orgânica. Além disso, concomitantemente à pesquisa com seres hu- 
manos, caminham os dilemas éticos envolvidos na pesquisa qualitativa em saúde, uma vez que esta se baseia nos métodos das ciências humanas, e não nos métodos experimentais biomédicos. Contudo, é necessário complementar que, muito mais que a diferença metodológica existente entre as ciências humanas e a biomedicina, estão presentes também as diferenças epistemológicas ${ }^{16}$.

Em face de tais diferenças e peculiaridades, aponta-se a necessidade de uma regulamentação específica para as ciências humanas que aborde os dilemas éticos envolvidos nas pesquisas epistemológicas, as quais são alheias ao modelo biomédico de pesquisa observado desde a Resolução 196/96. Diante disso, ressalta-se a relevância de se levantarem questões voltadas para a melhoria da análise das implicações éticas das pesquisas antropológicas e da elaboração de propostas de regulação de procedimentos éticos considerando os marcos da etnografia ${ }^{16}$.

Ainda sobre o exposto, acrescenta-se que essa regulamentação não deve se restringir à atividade de pesquisa no meio universitário ou em instituições de pesquisa, mas sim ser abrangente, incluindo aspectos voltados para as atividades de organizações não governamentais (ONG) e de órgãos públicos que promovem políticas sociais. Afinal, ainda que as ONG realizem pesquisas de caráter antropológico e façam uso dos mesmos instrumentos e técnicas de investigação, suas atividades raramente são supervisionadas pelos órgãos responsáveis pela observância da regulamentação ${ }^{17}$.

Figura 2. Instrumentos e/ou procedimentos de coleta de dados utilizados em pesquisas submetidas a um CEP do município de Divinópolis/MG, Brasil

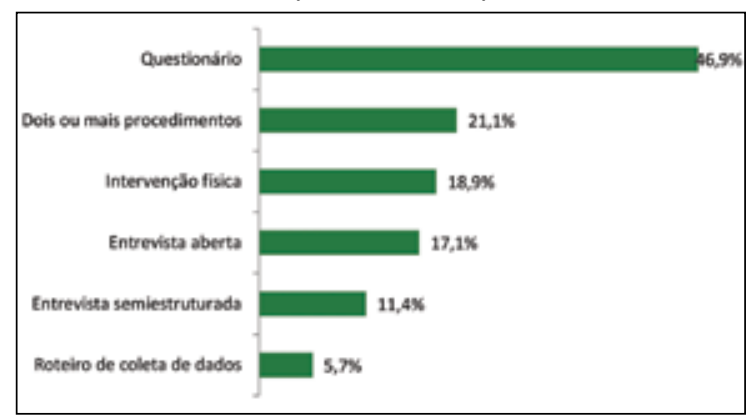

Tal como indicado na Figura 2, a maioria das pesquisas $(46,9 \%)$ foi realizada por meio da aplicação de questionários. É bastante comum o uso de entrevista e questionário em pesquisas de campo, sendo o questionário o instrumento mais utilizado para o levantamento de informações. Ele não precisa estar restrito a determinada quantidade de questões, porém aconselha-se que não seja muito extenso a fim de evitar o desânimo do pesquisado ${ }^{15}$. Cabe dizer que, entre os pesquisadores, 27 (21,1\%) optaram por coletar os dados utilizando dois tipos de procedimento ou mais, motivo pelo qual a soma das porcentagens na Figura 2 ultrapassa 100\%.

\section{Descrição de riscos no TCLE}

Em relação à categoria de descrição de riscos no TCLE, notou-se que, dos 175 pesquisadores, $117(66,8 \%)$ não descreveram os riscos no TCLE e $36(20,5 \%)$ descreveram. Infere-se que o fato de a maioria não ter descrito os riscos no TCLE pode estar associado à classificação da palavra "risco" pelos pesquisadores, considerando que ela pode "assustar" os participantes por ser muito "forte", dado que, muitas vezes, é associada a riscos físicos e à relação entre vida e morte. Tal consideração torna ainda mais relevante a exigência da descrição dos riscos em todos os projetos de pesquisa, no corpo do texto e também no TCLE, os quais devem ser anexados ao protocolo de pesquisa para avaliação pelo CEP/Conep ${ }^{18}$. Também se identificou nos protocolos solicitação de dispensa do TCLE em 17 dos projetos analisados $(9,7 \%)$, por se tratar de pesquisas documentais. Além disso, observou-se que 5 protocolos $(2,9 \%)$ não contavam com seus respectivos TCLE, por motivos desconhecidos.

\section{Descrição das medidas de precaução/prevenção dos riscos no TCLE}

Finalmente, sobre a exigência de descrição das medidas de prevenção/precaução no TCLE, observou-se que, do total de pesquisadores, 10 (6,5\%) não as descreveram e $143(93,5 \%)$ as descreveram direcionadas para a garantia de confidencialidade e sigilo, a retirada da participação da pesquisa a qualquer momento e a garantia de assistência psicológica, se necessária. Cabe lembrar que 17 pesquisadores solicitaram dispensa de TCLE e que 5 protocolos não traziam o TCLE anexado. A descrição dos riscos e benefícios decorrentes da participação de sujeitos em pesquisas, bem como das medidas de prevenção e proteção a eles destinadas, é inerente à função de pesquisador e própria de seu papel diante dos que participam dos experimentos. Em decorrência, advoga-se que tal descrição siga a prescrição do modelo de protocolo proposto pelo CEP.

\section{Considerações finais}

O desenvolvimento deste estudo permitiu verificar que os pesquisadores, embora tenham 
identificado, na maioria das vezes, as medidas de precaução/prevenção no protocolo de pesquisa e no TCLE, apresentaram dificuldade em descrever os riscos inerentes às suas pesquisas, tanto no protocolo quanto no TCLE. Chama a atenção o fato de boa parte dos pesquisadores não ter descrito os riscos de suas pesquisas pelo fato de utilizar questionários e/ou entrevistas como forma de coleta de dados. Cabe ressaltar que na Resolução 466/12 que prevê a possibilidade de se estabelecer norma específica para regular a ética nas pesquisas das ciências sociais, que normalmente utilizam técnicas classificadas como qualitativas - a descrição dos riscos é tida como obrigatória e, provavelmente, deverá ser mantida nas diretrizes propostas para essas áreas.

Nesse sentido, da mesma forma que existe distinção significativa em termos de metodologia entre pesquisa quantitativa e pesquisa qualitativa, há no bojo dessa última importantes diferenças relativas à técnica de levantamento de dados que precisam ser bem conhecidas e consideradas pelos pesquisadores. A rigor, aquilo que é chamado de pesquisa qualitativa envolve desde a aplicação de instrumentos de levantamento de dados, fechados ou semiestruturados, até a entrevista aberta e observação participante. A diferença no processo de obtenção, descrição e análise das informações em cada uma dessas formas de coleta de dados é muito significativa e precisa ser conhecida a fundo pelos pesquisadores, a fim de evitar a subsunção dos ris- cos aos participantes, ocultando-os sob definições amplas, consideradas inofensivas, como "pesquisa qualitativa" ou "aplicação de questionários".

Considerando que frequentemente o pesquisador lança mão de questionários e entrevistas em suas pesquisas, é de suma importância que ele conheça os possíveis riscos envolvidos em tais procedimentos, com vistas a adotar medidas de prevenção e proteção. Ressalta-se, portanto, a importância de promover capacitações de cunho interdisciplinar para os pesquisadores, a fim de que sejam esclarecidos acerca de todos os pontos da legislação ética brasileira referentes às pesquisas envolvendo seres humanos, bem como das especificidades das técnicas da pesquisa quantitativa e qualitativa, tanto nas ciências biológicas quanto nas ciências comportamentais - aquelas ligadas a psicologia e psiquiatria - e nas ciências oriundas das áreas sociais.

Além de proporcionar um momento favorável à reflexão ética conjunta, a capacitação interdisciplinar pode acelerar o processo de avaliação do projeto de pesquisa, já que os pesquisadores, devidamente esclarecidos, terão menos dificuldade em identificar os riscos e associá-los às medidas de precaução/prevenção e, consequentemente, a aprovação de seus projetos será mais rápida. $\mathrm{O}$ mais importante desse processo, contudo, é que esse conhecimento trará mais segurança aos participantes das pesquisas, uma vez que serão informados sobre os processos e procedimentos de maneira inequívoca.

A pesquisa relatada neste artigo foi financiada pelo Conselho Nacional de Desenvolvimento Científico e Tecnológico (CNPq).

\section{Referências}

1. Conselho Nacional de Saúde. Resolução no 466, de 12 de dezembro de 2012. Aprovar as diretrizes e normas regulamentadoras de pesquisas envolvendo seres humanos. [Internet]. 2012 [acesso 10 mar 2014]. Disponível: http://conselho.saude.gov.br/resolucoes/2012/Reso466.pdf

2. Hammerschmidt KSA, Borghi ACS, Lenardt MH. Ética e estética: envolvimentos na promoção do cuidado gerontológico de enfermagem. Texto Contexto Enferm. 2006;15(especial):114-24.

3. Marcolino JAM, Cohen C. Sobre a correlação entre a bioética e a psicologia médica. Rev Assoc Med Bras. 2008;54(4):363-8.

4. Cohen C. Por que pensar a bioética? Rev Assoc Med Bras. 2008;54(6):473-4.

5. Guerriero ICZ, Schmidt MLS, Zicker F, organizadores. Ética nas pesquisas em ciências humanas e sociais na saúde. São Paulo: Hucitec; 2008.

6. Araújo LZS. Aspectos éticos da pesquisa científica. Pesqui Odontol Bras. 2003;17 (Supl. 1):57-63.

7. Furukawa $P O$, Cunha ICKO. Comitês de ética em pesquisa: desafios na submissão e avaliação de projetos científicos. Rev Bras Enferm. 2010;63(1):145-7.

8. Cabral MML, Schindler HC, Abath FGC. Regulamentações, conflitos e ética da pesquisa médica em países em desenvolvimento. Rev Saúde Pública. 2006;4(3):521-7.

9. Sawaia BB. Limites do julgamento ético nos estudos que se valem de técnicas qualitativas. BIS Boletim do Instituto de Saúde. 2005;(35):22-4. 
10. Palácios M, Rego S, Schramm FR. A eticidade da pesquisa em seres humanos. Escola Nacional de Saúde Pública. Comitê de Ética em Pesquisa. [Internet]. Rio de Janeiro: ENSP; 2001 [acesso nov 2014]. Disponível: http://www4.ensp.fiocruz.br/etica/docs/artigos/Eticidade.pdf

11. Cardoso MVLML. Produção e divulgação de pesquisa em enfermagem. Rev Rene. 2012;13(2):252.

12. Fontelles MJ, Carvalho RM, D'Oliveira MS. Estudo analítico do Comitê de Ética em Pesquisa da Universidade da Amazônia. Rev Para Med. 2007;21(2):19-22.

13. Novaes MRCG, Guilhem D, Lolas F. Dez anos de experiência do Comitê de Ética em Pesquisa da Secretaria de Saúde do Distrito Federal, Brasil. Acta Bioeth. 2008;14(2): 185-92.

14. Massarollo MCKB, Kurcgant P, Fernandes MFP. Comitê de Ética em Pesquisa da Escola de Enfermagem da USP: experiência dos primeiros seis anos. Rev Esc Enferm USP. 2009;43(especial 2):1303-7.

15. Pessalacia JDR. Bioética e pesquisa: percepção dos sujeitos de pesquisa acerca de assuntos e situações constrangedoras em pesquisas com questionamentos [tese]. Ribeirão Preto: Universidade de São Paulo/Escola de Enfermagem de Ribeirão Preto; 2009.

16. Sarti C, Duarte LFD. Antropologia e ética: desafios para a regulamentação. Brasília: ABA; 2013.

17. Fleischer $S$, Schuch $P$, organizadores. Ética e regulamentação na pesquisa antropológica. Brasília: Letras Livres; 2010.

18. Teixeira RP, Nunes MLT. A utilização do consentimento informado em psicoterapia. Rev Aletheia. 2007;(26):137-45.

\section{Participação das autoras}

Camila Maria Pereira Rates realizou a coleta de dados, redação e formatação final do artigo. Marcella Rodrigues e Costa realizou a coleta de dados e redação do artigo. Juliana Dias Reis Pessalacia orientou a pesquisa e contribuiu para a redação do artigo.

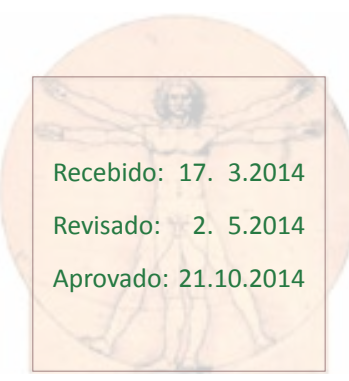

